\title{
Phenotypic consequences of red-white colony type variation in Mycobacterium avium
}

\author{
Gerard A. Cangelosi, ${ }^{1}$ Christine O. Palermo ${ }^{1}$ and Luiz E. Bermudez ${ }^{2}$ \\ Author for correspondence: Gerard A. Cangelosi. Tel: +1 206284 8846. Fax: +1 2062840313. \\ e-mail: gcang@sbri.org
}

\footnotetext{
1 Seattle Biomedical Research Institute, 4 Nickerson Street, WA 98109, Seattle, USA

2 Kuzell Institute of Arthritis and Infectious Disease, San Francisco, CA, USA
}

\begin{abstract}
Mycobacterium avium undergoes reversible morphotypic switching between the virulent transparent colony type and the less virulent opaque colony type. A new morphotypic switch in $M$. avium, termed red-white, that becomes visible when opaque colonies of clinical isolates are grown on agar media containing Congo red, was recently described. White opaque (WO) variants were found to be more resistant to multiple antibiotics than were red opaque (RO) variants. The present paper reports that transparent derivatives of RO and WO clones retain the differential Congo red binding properties of their opaque parents, indicating that the opaque-transparent switch operates independently of the red-white switch. White transparent variants were more resistant to clarithromycin and rifampin in vitro, and better able to survive within human macrophages, than their red transparent counterparts. Neither red nor white variants were markedly favoured during growth in vitro; however, red variants were better able to spread on soft agar (sliding motility), a potential selective advantage under some environmental circumstances. White-to-red switching was frequently observed in vitro and was accompanied by decreased antibiotic resistance and increased motility. Red-to-white switching has yet to be observed in vitro, indicating that the red morphotype is very stable. Significantly, some widely studied laboratory reference strains of $M$. avium, including strain 2151 and the genome sequence strain 104, are stable red clones. These strains are intrinsically antibiotic resistant and virulent in animal models, but they may not express genes encoding the elevated levels of antibiotic resistance and intracellular survival observed in white variants.
\end{abstract}

Keywords: AIDS, drug resistance, virulence, motility, phenotypic switching

\section{INTRODUCTION}

Mycobacterium avium is an environmental organism and a common opportunistic pathogen of AIDS patients and other susceptible individuals. Most M. avium isolates segregate into smooth-transparent-, smoothopaque- and rough-colony-type variants on agar plates. Transparent variants predominate in patient samples and grow better in macrophage and animal models. Opaque variants usually grow better on laboratory media and typically predominate after repeated passage (Belisle \& Brennan, 1994; Inderlied et al., 1993; Inderlied

Abbreviations: $\mathrm{CR}$, Congo red; $\mathrm{DR}$, dark red; RCRB, relative Congo red binding; RO, red opaque; RT, red transparent; WO, white opaque; WT, white transparent; ZOI, zone of inhibition.
\& Nash, 1996; Prinzis et al., 1994). Thus, the opaquetransparent switch may help the bacterium to survive and flourish under diverse conditions. Rough variants also appear after passage in vitro; however, conditions favouring their proliferation have not been identified. The opaque-transparent switch is reversible at frequencies of $10^{-4}$ to $10^{-6}$ per generation, but the rough-colony type results from irreversible deletion of cell envelope glycopeptidolipid genes (Belisle et al., 1993; Inderlied et al., 1993; Prinzis et al., 1994; Woodley \& David, 1976).

M. avium is intrinsically resistant to many drugs used to treat other mycobacterial infections. This resistance is usually ascribed to the impermeability of its lipid-rich cell wall, but it may also occur through other mechanisms (Inderlied et al., 1993; Jarlier \& Nikaido, 
1994; Mdluli et al., 1998; Peloquin, 1997; PortilloGomez et al., 1995). Effective drugs such as clarithromycin, rifabutin, fluoroquinolones and amikacin must be administered in combination to prevent the possible emergence of drug resistance. For unknown reasons, transparent variants are more drug-resistant than opaque variants. Therefore, laboratory drug-susceptibility testing must be carried out on isolated transparent colonies. However, even with this precaution there is considerable assay-to-assay variability, suggesting that additional morphotypic switches may also affect drug susceptibility (Heifets, 1996; Inderlied \& Nash, 1996; Sison et al., 1996).

We recently described a new phenotypic variation detectable among opaque colonies of $M$. avium grown on agar media containing the lipoprotein stain Congo red (CR). About one-third of clinical isolates examined formed red opaque ( $\mathrm{RO}$ ) as well white opaque (WO) colonies under these conditions, while the remaining two-thirds formed only red and, in some cases, pink opaque colonies (Cangelosi et al., 1999). RFLP analysis using an IS1245 probe showed that the RO and WO variants were segregants of single strains, not distinct strains. WO clones were found to be more resistant to antimycobacterial drugs than were their $\mathrm{RO}$ counterparts. The physiological basis for red-white variation has not yet been determined.

In contrast to opaque colonies, which were vividly heterogeneous on CR plates, transparent colonies of clinical isolates had uniform pale pink colours that did not differ much from the underlying CR agar. For this reason, our previous study did not reveal whether red-white segregation also exists within the more clinically significant transparent morphotype. The present report shows that it does. Transparent derivatives of WO clones (WT) bound less CR, were more resistant to multiple antibiotics and were better able to survive within human macrophages than were transparent derivatives of RO clones (RT). We also examined the stability of the white and red morphotypes and the effects of red-white switching on growth and spreading in vitro.

\section{METHODS}

Bacterial strains and culture media. The M. avium clinical isolates and laboratory strains used in this study are listed in Table 1 . Bacteria were grown at $37^{\circ} \mathrm{C}$ on Middlebrook $7 \mathrm{H} 10$ agar (Difco-Becton Dickinson) containing OADC enrichment and $0.5 \%$ glycerol (MAG) unless stated otherwise. CR was added to agar media to $100 \mu \mathrm{g} \mathrm{ml}^{-1}$ (MAG-CR) and stored as described (Cangelosi et al., 1999).

Isolation of transparent phase variants. RT and WT phase variants of strain HMC02 were isolated directly from RO and WO parents by selection on ciprofloxacin E-test plates, as follows. Opaque colonies picked from MAG-CR plates were suspended in Middlebrook $7 \mathrm{H} 9$ broth and then spread onto fresh MAG-CR plates. Ciprofloxacin E-test strips (Remel Microbiology Products) were applied aseptically. Plates were incubated in polyethylene bags at $37^{\circ} \mathrm{C}$ under $5 \% \quad \mathrm{CO}_{2}$.
Transparent colonies, distinguishable from opaque colonies by stereoscopic microscopy with transmitting light (Belisle \& Brennan, 1994), began to appear within the opaque zones of inhibition (ZOIs) after 5 weeks incubation. Three transparent colonies derived from the RO parent were isolated and designated HMC02-RT1, -RT2, -RT3, and three transparent colonies derived from the WO parent were isolated and designated HMC02-WT1, -WT2, -WT3. Transparent colonies growing closest to the ' $32 \mu \mathrm{g} \mathrm{ml}^{-1}$, mark on the E-test strip were picked in both cases.

Transparent phase variants of strain HMC10 were isolated without antibiotic selection, as follows. RO and WO cells grown on MAG plates were suspended in autoclaved deionized water to a density of $10^{7}$ c.f.u. $\mathrm{ml}^{-1}$. The suspensions were incubated without agitation at room temperature for $4 \mathrm{~d}$ and then spread onto MAG-CR plates to obtain isolated colonies. Following this procedure, transparent colonies accounted for $1-30 \%$ of total colonies, compared to $<1 \%$ prior to incubation in water (data not shown). Three transparent clones derived from the RO parent were subcultured onto MAG-CR plates and examined by stereoscopic microscopy for light transmission to confirm their stable conversion to the transparent phase. These clones were designated HMC10-RT1, -RT2 and -RT3. Three transparent clones derived from the WO parent, HMC10-WT1, -WT2 and -WT3, were isolated by the same process.

In vitro drug susceptibility tests. For comparing the drug susceptibilities of isolated colony-type variants, we have found that agar diffusion assays give more consistent results than broth-based susceptibility tests because colony morphotypes are clearly visible on agar plates, and spurious results arising from morphotypic switching are thereby avoided. Accordingly, clarithromycin and rifampin susceptibilities were assessed by using disc-diffusion assays. Bacteria were spread onto MAG-CR plates, then filter paper discs impregnated with $15 \mu \mathrm{g}$ clarithromycin (Remel Microbiology Products) or $25 \mu \mathrm{g}$ rifampin (BBL, Difco-Becton Dickinson) were placed onto the plates. Disc-diffusion plates were incubated in polyethylene bags for $14 \mathrm{~d}$ at $37{ }^{\circ} \mathrm{C}$ under $5 \% \mathrm{CO}_{2}$ before ZOIs of growth around the discs were measured.

Isolation of human macrophages. Monocytes were isolated by Histopagne-1077 (Sigma) gradient centrifugation from the white cell concentrate (Blood Center of the Pacific, San Francisco, CA). Mononuclear phagocytes were obtained as previously described (Bermudez et al., 1997; Hsu et al., 1995). Briefly, non-adherent cells were removed by washing after $2 \mathrm{~h}$ incubation in RPMI-1640 medium (Gibco-BRL, Life Technologies) supplemented with $10 \%$ fetal bovine serum and $2 \mathrm{mM}$ L-glutamine (Sigma). Adherent mononuclear phagocytes differentiate into macrophages after $4 \mathrm{~d}$ in culture.

Infection of human macrophages. Macrophage monolayers $\left(5 \times 10^{5}\right.$ cells) were incubated for $1 \mathrm{~h}$ with $\mathrm{RO}$, WO, RT or WT clones of strains HMC02 and HMC10, at a ratio of 10 bacteria per macrophage. Extracellular bacteria were removed from the monolayers by two gentle washes with HBSS warmed to $37^{\circ} \mathrm{C}$. Infected macrophages were incubated in fresh RPMI1640 medium at $37^{\circ} \mathrm{C}$ under $5 \% \mathrm{CO}_{2}$. To measure resistance to killing and inhibition of growth by macrophages, the number of intracellular bacteria $4 \mathrm{~d}$ after infection was compared to the number of intracellular bacteria $2 \mathrm{~h}$ after infection. Intracellular bacteria were harvested after lysis of the macrophages as previously described (Bermudez et al., 1997; Hsu et al., 1995). Lysates were serially diluted and plated onto $7 \mathrm{H} 11$ agar plates and incubated at $37^{\circ} \mathrm{C}$. c.f.u. were counted after $10 \mathrm{~d}$. 
Table 1. M. avium isolates and variants used in this study

\begin{tabular}{|c|c|c|}
\hline Isolate* & $\begin{array}{l}\text { Red-white } \\
\text { morphotype }\end{array}$ & Comments \\
\hline HMC02 & Mixed $\dagger$ & Clinical isolate (Cangelosi et al., 1999) \\
\hline HMC02-WO & White & Isolated from HMC02 \\
\hline HMC02-WT2 & White & Derived from HMC02-WO \\
\hline HMC02-WT3 & White & Derived from HMC02-WO \\
\hline HMC02-RO & Red & Isolated from HMC02 \\
\hline HMC02-RT2 & Red & Derived from HMC02-RO \\
\hline HMC02-RT3 & Red & Derived from HMC02-RO \\
\hline HMC10 & Mixed $\dagger$ & Clinical isolate (Cangelosi et al., 1999) \\
\hline HMC10-WO & White & Isolated from HMC10 \\
\hline HMC10-WT1 & White & Derived from HMC10-WO \\
\hline HMC10-WT2 & White & Derived from HMC10-WO \\
\hline HMC10-WT3 & White & Derived from HMC10-WO \\
\hline HMC10-RO & Red & Isolated from HMC10 \\
\hline HMC10-RT1 & Red & Derived from HMC10-RO \\
\hline HMC10-RT2 & Red & Derived from HMC10-RO \\
\hline HMC10-RT3 & Red & Derived from HMC10-RO \\
\hline HMC04 & Red & Clinical isolate (Cangelosi et al., 1999) \\
\hline 102 & Mixed $\dagger$ & $\begin{array}{l}\text { Laboratory strain kindly provided by Clark Inderlied, Children's Hospital, Los } \\
\text { Angeles (Bermudez et al., 1999) }\end{array}$ \\
\hline 104 & Red & $\begin{array}{l}\text { Laboratory strain kindly provided by William Bishai, Johns Hopkins } \\
\text { University; source of DNA for genomic sequencing by The Institute for } \\
\text { Genomic Research (Bermudez et al., 1991) }\end{array}$ \\
\hline 2151 & Red & $\begin{array}{l}\text { Laboratory strain kindly provided by Julia Inamine, Colorado State University } \\
\text { (Belisle \& Brennan, 1994; Belisle et al., 1993; Martinez et al., 1999) }\end{array}$ \\
\hline
\end{tabular}

*All isolates formed opaque, transparent and rough variants. Isolates designated ' $\mathrm{O}$ ' were predominantly opaque, and isolates designated ' $\mathrm{T}$ ' were predominantly transparent.

†'Mixed' clinical isolate and laboratory stocks, when plated onto CR agar, formed both red and white colonies.

Sliding motility. RO, WO, RT and WT clones were picked from frozen stocks or from actively growing (10-d-old) colonies on MAG-CR plates. Using a $1 \mu$ l loop, bacteria were inoculated into the centre of $100 \times 15 \mathrm{~mm}$ plates containing MAG or MAG-CR semi-solidified with $0.3 \%$ agar (Martinez et al., 1999). Plates were incubated in sealed polyethylene bags at $37^{\circ} \mathrm{C}$ under $5 \% \mathrm{CO}_{2}$. Spreading was measured at various time points over the course of $2-4$ weeks.

\section{RESULTS}

\section{Isolation of RT and WT variants}

Our previous study focused on opaque variants because of their vivid heterogeneity on CR plates (Cangelosi et al., 1999). By contrast, transparent colonies of clinical isolates were nearly uniform on CR plates, appearing pale translucent pink. To determine whether red-white heterogeneity is possible within the transparent morphotype, we isolated transparent derivatives of readily distinguishable RO and WO clones of strains HMC02 and HMC10 and asked whether they retained characteristics that distinguish their $\mathrm{RO}$ and $\mathrm{WO}$ parents. Transparent derivatives of HMC02-RO and -WO were isolated by ciprofloxacin selection, and transparent derivatives of HMC10-RO and -WO were isolated after incubation in deionized water. As shown in Fig. 1, all transparent derivatives retained the differential CRbinding properties of their opaque progenitors, as determined by using the relative Congo red binding (RCRB) assay described previously (Cangelosi et al., 1999). Therefore, at least some characteristics that distinguish the red and white morphotypes were unaffected by the opaque-to-transparent switch. Accordingly, we assigned the terms 'red transparent' (RT) and 'white transparent' (WT) to transparent derivatives of $\mathrm{RO}$ and $\mathrm{WO}$ clones, respectively.

Distinctions between RT and WT variants also survived the switch back to the opaque morphotype. When grown as lawns on MAG agar, RT and WT clones of strains HMC02 and HMC10 formed opaque colonies at high frequencies consistent with past reports (Woodley \& David, 1976). Opaque derivatives of RT clones were uniformly RO or dark red (DR). Opaque derivatives of WT clones were more diverse and included WO, RO, pink and DR colonies. The ability of WT clones to switch to multiple morphotypes is shared by WO clones, as described later in this report. These observations suggest that opaque-transparent variation and redwhite variation are independent phenomena. 


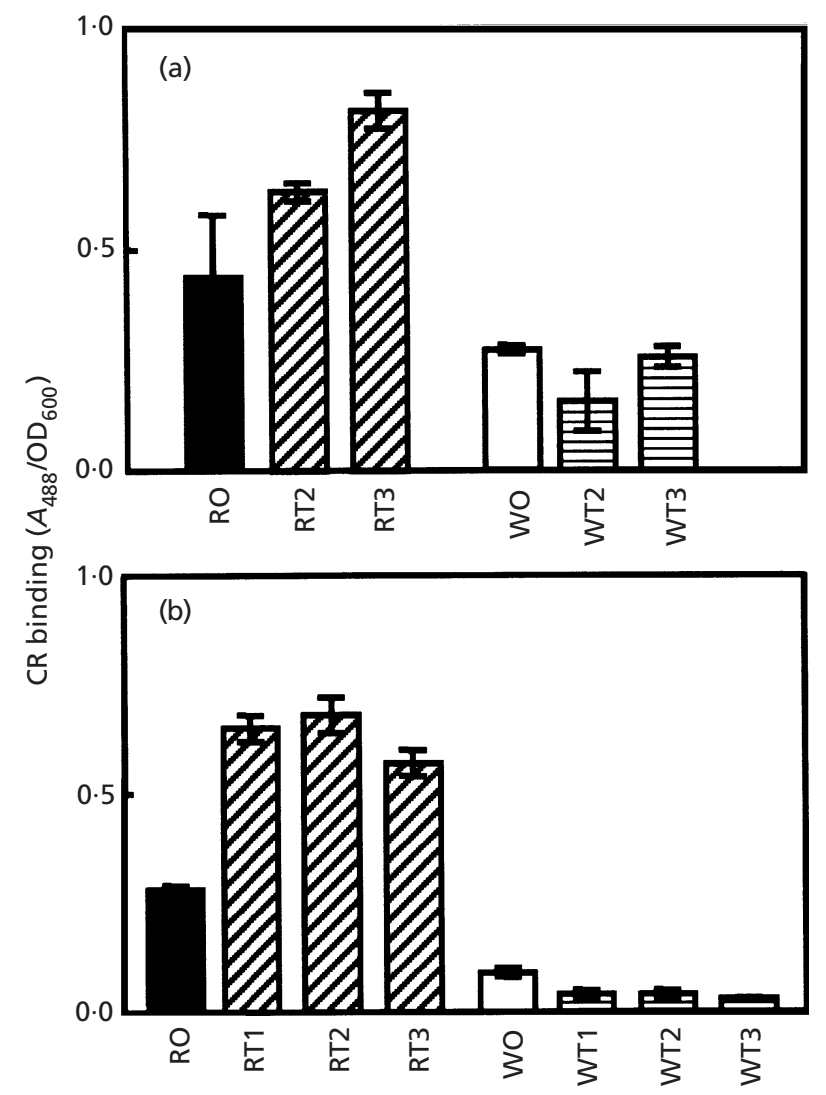

Fig. 1. $C R$ binding by $W O$ and $R O$ clones of strains $\mathrm{HMCO2}$ (a) and HMC10 (b), and by their WT and RT derivatives. CR binding normalized to cell numbers was measured by using the relative Congo red binding (RCRB) assay described previously (Cangelosi et al., 1999). Means and standard deviations of three measurements are shown. In this and subsequent figures, bars representing RO variants are black, bars representing WO variants are white, bars representing RT variants are diagonally striped, and bars representing WT variants are horizontally striped.

\section{RT and WT variants differ with regard to drug susceptibility}

Drug susceptibility of transparent clones was measured by agar diffusion. As in our previous study (Cangelosi et al., 1999), this approach gave more reproducible results than broth-based susceptibility tests because colony morphotypes were clearly visible on plates. In both HMC02 and HMC10, WT clones were significantly more resistant than their RT counterparts to clarithromycin and rifampin (Fig. 2). Therefore, WT clones of both strains retained the multidrug-resistance properties of their WO progenitors.

\section{RT and WT variants differ with regard to intracellular growth}

It has long been known that transparent variants are better able to survive and grow within macrophages than are opaque variants (Inderlied et al., 1993). To
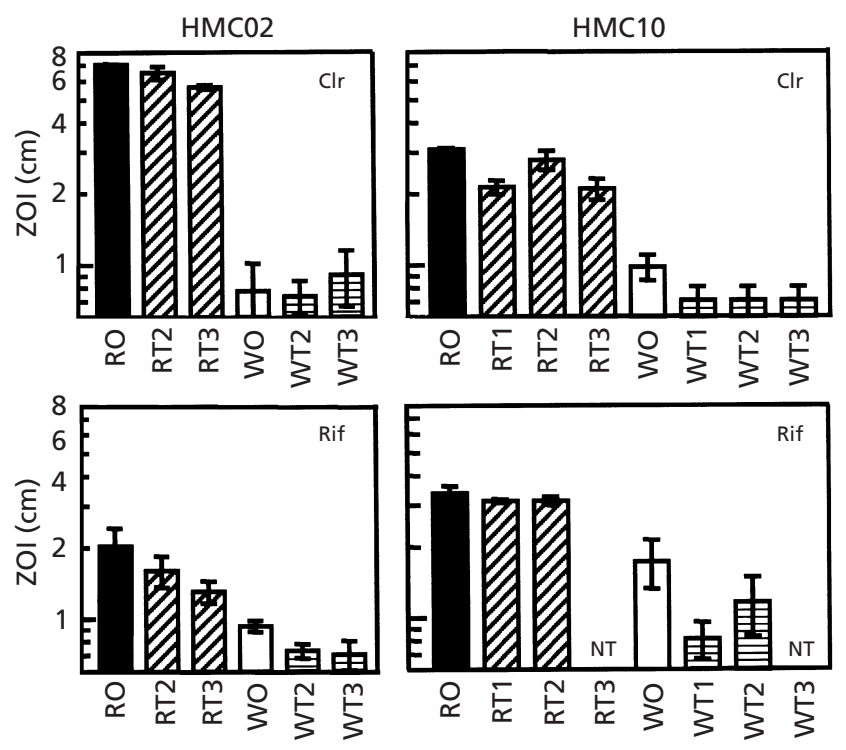

Fig. 2. Susceptibility of WO, RO, WT and RT clones of strains HMCO2 (left) and HMC10 (right) to clarithromycin (Clr) and rifampin (Rif). Susceptibilities were determined by disc diffusion as described in Methods. Zones of inhibition (ZOIs) in $\mathrm{cm}$ are shown; smaller values correspond to higher levels of resistance. Means and standard deviations of three experiments are shown. NT, not tested.

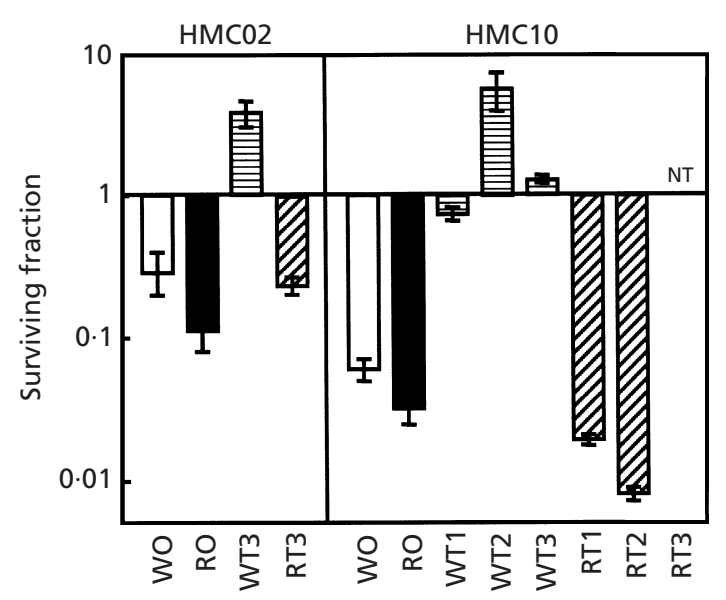

Fig. 3. Survival of WO, RO, WT and RT clones within human macrophages $4 \mathrm{~d}$ after infection. The surviving fraction is the number of c.f.u. per unit cell culture volume measured at day 4 divided by the same value measured at day 0 . Means and standard deviations of three experiments are shown. NT, not tested.

determine whether the red-white switch also affects this disease-related phenotype, RO, WO, RT and WT clones of strains HMC02 and HMC10 were tested for their ability to survive and grow within human macrophages. WT clones of both strains survived and in some cases grew under these conditions, while viability of RT, $\mathrm{RO}$ and $\mathrm{WO}$ clones decreased after $4 \mathrm{~d}$ (Fig. 3). Thus 


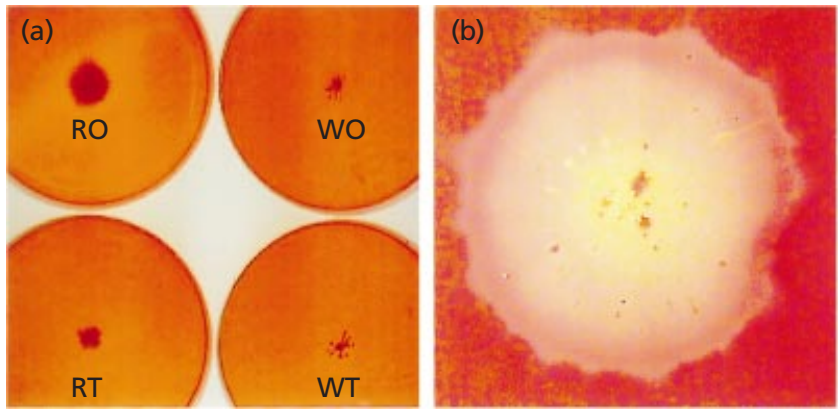

Fig. 4. Spreading of $M$. avium morphotypic variants on $0.3 \%$ agar plates containing CR. (a) Back-lit photograph of HMCO2RO, -WO, -RT3 and -WT3 colonies taken $17 \mathrm{~d}$ after inoculation. Similar results were observed in HMC10. (b) Front-lit photograph of an HMC10-WO colony taken $23 \mathrm{~d}$ after inoculation.

red-white segregation affects resistance to killing by human macrophages.

\section{Effects of red-white variation on extracellular growth phenotypes}

We noted previously that $\mathrm{RO}$ variants dispersed more easily in broth than did WO variants (Cangelosi et al., 1999). The same was true of RT clones isolated for this report relative to their WT counterparts. This difference did not translate to significant growth advantages for either morphotype during cultivation on Middlebrook agar plates or broth. RO variants of some strains formed larger colonies than their WO counterparts on agar plates; however, RO variants did not overgrow WO variants when mixed broth cultures were passed eight times $(10 \%, \mathrm{v} / \mathrm{v}$, inocula) over the course of 18 weeks.

The two morphotypes differed with regard to sliding motility on soft agar. Martinez et al. (1999) recently published the first detailed report of sliding motility in Mycobacterium species, including $M$. avium. Sliding motility was defined as the ability of cell masses to spread over moist surfaces as cell numbers increase. To determine whether red-white variation affects sliding motility, we inoculated $0.3 \%$ agar plates with RO, WO, RT and WT clones of strains HMC02 and HMC10 as described by Martinez et al. (1999). All variants sometimes developed clear haloes that contained few or no bacterial cells detectable by cultivation. These haloes may have been composed of extracellular material that diffused through the agar. Within the haloes, colonies of red and white variants exhibited different patterns of spreading. As shown in Fig. 4a, red variants initially spread more aggressively than their white counterparts. For the first 2-3 weeks of each experiment, WO and WT clones appeared as confined, non-motile colonies with 'branches' that may have spread through small cracks in the agar at the inoculation site. By contrast, RO and RT clones exhibited the distinctive spreading patterns described by Martinez et al. (1999). RO colonies spread more quickly than did RT colonies. Similar results were observed whether inocula were frozen cell stocks or actively growing cells picked from MAG-CR plates, suggesting that results were not influenced by varying physiological states of the inocula. The presence or absence of CR in the soft agar also did not affect results.

After extended incubation ( $>3$ weeks), white colonies sometimes began to spread more aggressively. When observed on soft agar containing CR, it was evident that cells in the spreading zones had lost the white phenotype, becoming pink or red in appearance (Fig. $4 \mathrm{~b}$; the isolated deep red sectors are discussed later). We concluded that a switch to a motile red morphotype had occurred, allowing spreading to begin. These observations showed that the red and white morphotypes differ in their abilities to spread over the surface of soft agar plates, with the red morphotype enjoying an advantage in this regard.

\section{Directionality of red-white switching}

In our previous study (Cangelosi et al., 1999), we plated stocks of 15 clinical isolates of $M$. avium onto CR agar and reported their staining phenotypes. Prior to plating onto CR agar, these stocks had been passed three to four times on Middlebrook 7H11 agar and LowensteinJensen slants, and some of them had also been cultured once on BACTEC broth. Of the 15 isolates, five formed 'mixed' (RO and WO) colonies, while the remaining ten formed red and/or pink colonies, but no white colonies. Since that study, we have examined some isolates in greater detail to determine the stability of the red and white phenotypes in vitro. Isolated RO clones of ' mixed' strains HMC02 and HMC10 were very stable, forming red, pink, or DR colonies but no white colonies over the course of repeated transfers. Pink and DR variants were not characterized further. $\mathrm{RO}$ clones isolated from a 'mixed' laboratory strain, 102, also formed no white colonies. Attempts to use antibiotic selection to isolate white derivatives of HMCO2-RO were unsuccessful. Strains that formed only RO and RT colonies, such as the clinical isolate HMC04 and laboratory strains 104 and 2151, were similarly stable, forming no white colonies after repeated passage on broth and agar media. All of these strains switched back and forth between the opaque and transparent morphotypes at typical frequencies (Woodley \& David, 1976).

In contrast to red variants, plates spread thickly with isolated WO clones frequently formed $\mathrm{RO}$ colonies as well as pink and DR colonies. The latter are visible as isolated deep red sectors in Fig. 4(b). RO derivatives of HMC02-WO were isolated and found to be as stable as any other RO clone. When ciprofloxacin susceptibility was measured by using E-tests as described previously (Cangelosi et al., 1999), these RO derivatives were approximately 10 -fold more susceptible than their WO parent, similar to other RO variants of HMCO2 (data not shown).

The relative stability of the red morphotype was especially evident when RT and WT variants of HMC02 and HMC10 were allowed to switch back to the opaque 
morphotype. Opaque derivatives of RT clones were never white, whereas those of WT clones were white, red, pink and DR. These observations indicate that the red morphotype is more stable than the white morphotype in vitro.

\section{DISCUSSION}

The data reported here indicate that red-white variation and opaque-transparent variation are independent phenomena. Because of this independence, it was possible to isolate and characterize RO, WO, RT and WT colony types of two clinical strains.

WT variants were significantly more resistant in vitro to two structurally unrelated drugs, clarithromycin and rifampin, than were their RT counterparts. In the agar diffusion assays we used, the difference between the red and white morphotypes matched or exceeded the difference between the opaque and transparent morphotypes. WT clones also resisted killing by human macrophages, which suggested that they are virulent and capable of contributing to treatment failure by virtue of their drug resistance. The relative sensitivity of RT clones to killing by macrophages does not necessarily indicate that the red morphotype is avirulent. Twothirds of the $M$. avium clinical isolates we examined previously did not form white colonies (Cangelosi et al., 1999), and strains 104 and 2151, which are virulent in animal models, formed only red colonies when plated on CR agar. Therefore, red variants probably retain at least some virulence properties. Nevertheless, the enhanced survival of WT variants within macrophages may reflect a higher level of pathogenicity. This possibility warrants further analysis using animal models of infection.

Sliding motility is a recently described property of mycobacteria, including M. avium (Martinez et al., 1999). The ability to spread over surfaces probably confers a selective advantage on environmental bacteria like $M$. avium, especially when nutrients become limiting locally. It was in this characteristic that the red morphotype appeared to have an advantage over the white morphotype. This was especially apparent upon extended incubation of white colonies on soft agar plates containing CR. Such colonies appeared white at the centre, surrounded by a spreading halo of red cells, suggesting that a switch to the red morphotype allowed cells to escape from the inoculation site.

White-to-red switching was observed not only on soft agar, but also on standard (1.5\% agar) CR plates inoculated with WO or WT cells. By contrast, we have yet to observe red-to-white switching in vitro. It was interesting that despite this strong disequilibrium in favour of the white-to-red switch in vitro, white variants were found in multiple clinical isolates (Cangelosi et al., 1999). The white morphotype may be favoured at some stage of infection, disease or treatment. Alternatively, red-to-white switching may be more common in vivo than in vitro. Examination of large numbers of clinical isolates before passage on laboratory media will help to refine these models, especially if the occurrence of white variants can be tested for association with higher rates of treatment failure.

WT clones switched to a variety of colony types, including WO, RO, DR, pink opaque and rough colonies. These colony types were diverse in size as well as in CR staining. Colonies formed by RT clones appeared much more uniform. This may explain why stable red clones such as 2151 and 104 have become widely studied laboratory strains. Strain 104 was the source of DNA for genomic sequencing of M. avium, which is nearing completion by The Institute for Genomic Research (http://www.tigr.org/tdb/mdb/ mdb.html). Strain 2151 has been the subject of extensive analysis of cell wall biochemistry (Belisle et al., 1993) and other characteristics including motility (Martinez et al., 1999 found strain 2151 to be motile like our red clones). As already noted, transparent variants of these strains are virulent and intrinsically drug resistant. However, they appear to be fixed in a form (red) that is less virulent and less drug resistant than the white form. Analysis of white variants may help us to better understand the full range of $M$. avium phenotypes that affect pathogenicity, drug resistance and survival in the environment.

\section{ACKNOWLEDGEMENTS}

We thank Dr Jean-Pierre Laurent for isolating some of the clones used in this study and Dr Nancy Freitag for critical reading of the manuscript. This work was supported by grants AI46226 and AI25767 from The National Institutes of Health, grant G8E10521 from The Environmental Protection Agency, and by The M. J. Murdock Charitable Trust. C. O.P. received support from the Mary Gates Endowment for Undergraduate Research and the NASA Space Grant Consortium.

\section{REFERENCES}

Belisle, J. T. \& Brennan, P. J. (1994). Molecular basis of colony morphology in Mycobacterium avium. Res Microbiol 145, 237-242.

Belisle, J. T., Klaczkiewicz, K., Brennan. P. J., Jacobs, W. R., Jr \& Inamine, J. M. (1993). Rough morphological variants of $\mathrm{Myco-}$ bacterium avium: characterization of genomic deletions resulting in the loss of glycopeptidolipid expression. J Biol Chem 268, 10517-10523.

Bermudez, L. E., Petrofsky, M., Kolonoski, P. \& Young, L. S. (1991). An animal model of Mycobacterium avium complex disseminated infection after colonization of the intestinal tract. $J$ Infect Dis 165, 75-79.

Bermudez, L. E., Parker, A. \& Goodman, J. (1997). Growth within macrophages increases the efficiency of Mycobacterium avium to invade human macrophages by a complement receptor-independent pathway. Infect Immun 65, 1916-1922.

Bermudez, L. E., Kolonoski, P., Wu, M., Aralar, P. A., Inderlied, C. B. \& Young, L. S. (1999). Mefloquine is active in vitro and in vivo against the Mycobacterium avium complex. Antimicrob Agents Chemother 43, 1870-1874.

Cangelosi, G. A., Palermo, C. O., Laurent, J.-P., Hamlin, A. M. \& Brabant, W. H. (1999). Colony morphotypes on Congo red agar segregate along species and drug susceptibility lines in the 
Mycobacterium avium-intracellulare complex. Microbiology 145, 1317-1324.

Heifets, L. (1996). Susceptibility testing of Mycobacterium avium complex isolates. Antimicrob Agents Chemother 40, 1759-1767.

Hsu, N., Young, L. \& Bermudez, L. E. (1995). Response to stimulation with recombinant cytokines and synthesis of cytokines by murine intestinal macrophages infected with Mycobacterium avium complex. Infect Immun 63, 528-534.

Inderlied, C. B. \& Nash, K. A. (1996). Microbiology and in vitro susceptibility testing. In Mycobacterium avium Complex Infection: Progress in Research and Treatment, pp. 100-140. Edited by J. A. Korvick \& C. A. Benson. New York: Marcel Dekker.

Inderlied, C. B., Kemper, C. A. \& Bermudez, L. E. M. (1993). The Mycobacterium avium complex. Clin Microbiol Rev 6, 266-310.

Jarlier, V. \& Nikaido, H. (1994). Mycobacterial cell wall: structure and role in natural resistance to antibiotics. FEMS Microbiol Lett 123, 11-18.

Martinez, A., Torello, S. \& Kolter, R. (1999). Sliding motility in mycobacteria. J Bacteriol 181, 7331-7338.

Mdluli, K., Swanson, J., Fischer, E., Lee, R. E. \& Barry, C. E. (1998). Mechanisms involved in the intrinsic isoniazid resistance of Mycobacterium avium. Mol Microbiol 27, 1223-1233.
Peloquin, C. A. (1997). Mycobacterium avium complex infection: pharmacokinetic and pharmacodynamic considerations that may improve clinical outcomes. Clin Pharmacokinet 32, 52-144.

Portillo-Gomez, L., Nair, J., Rouse, D. A. \& Morris, S. L. (1995). The absence of genetic markers for streptomycin and rifampicin resistance in Mycobacterium avium complex strains. J Antimicrob Chemother 36, 1049-1053.

Prinzis, S., Rivoire, B. \& Brennan, P. J. (1994). Search for the molecular basis of morphological variation in Mycobacterium avium. Infect Immun 62, 1946-1951.

Sison, J. P., Yao, Y., Kemper, C. A., Hamilton, J. R., Brummer, E., Stevens, D. A. \& Deresinski, S. C. (1996). Treatment of Mycobacterium avium complex infection: do the results of the in vitro susceptibility tests predict therapeutic outcome in humans? J Infect Dis 173, 677-683.

Woodley, C. L. \& David, H. L. (1976). Effect of temperature on the rate of transparent to opaque colony type transition in Mycobacterium avium. Antimicrob Agents Chemother 9, 113-119.

Received 20 June 2000; revised 11 September 2000; accepted 14 November 2000. 